\title{
ORIGINAL ARTICLE Management of cryptococcal meningitis in adults at Mthatha Hospital Complex, Eastern Cape, South Africa
}

O O Sogbanmu, ${ }^{1}$ MB ChB, PgDip (HIVMgt), MMed (Fam Med);

M A John, ${ }^{2,3}$ MB ChB, FCPath (Micro), Dip HIV Man (SA), DTMH, Cert Infectious Disease (SA);

U Lalloo, ${ }^{2} \mathrm{MB}$ ChB, FCP, DOH, MD

${ }^{1}$ Cecilia Makiwane Hospital, Eastern Cape Department of Health, East London, South Africa

${ }^{2}$ Enhancing Care Initiative, Nelson R Mandela School of Medicine, University of KwaZulu-Natal, Durban, South Africa

${ }^{3}$ Infectious Diseases Department, Nelson R Mandela School of Medicine, University of KwaZulu-Natal, Durban, South Africa

Corresponding author: M A John (johnm@ukzn.ac.za)

\begin{abstract}
Background. Cryptoccocal meningitis (CM) remains prevalent in HIV-infected individuals across South Africa (SA). Early diagnosis and management, aided by the existing Southern African HIV Clinicians Society (SAHIVSoc) 2007 guidelines on management of CM, could reduce the mortality associated with this condition.

Objective. To review the management of adult patients with CM and adherence to the SAHIVSoc 2007 guidelines in a district hospital. Methods. A retrospective chart review of patients admitted with CM from December 2011 to May 2012 was performed. The following key recommendations of the guidelines were evaluated: measurement of cerebrospinal fluid (CSF) opening pressure at the first lumbar puncture (LP), prescription of amphotericin B (AMB)/fluconazole therapy, intravenous prehydration preceding administration of AMB, monitoring of renal function and performance of serial LPs to manage raised intracranial pressure (ICP). Results. A total of 57 patient charts were reviewed, of which 40 (70\%) were of females. The mean age (range) of the cohort was $36(21-60)$ years. Fifty-two (91\%) patients presented with headache. Confusion was recorded in $30(53 \%)$ and vomiting in 26 (46\%). The major signs observed were fever $(n=29(51 \%))$ and neck stiffness $(n=34(60 \%))$. Fifty-five $(96 \%)$ patients were HIVinfected at presentation, with a median (range) $\mathrm{CD}^{+}$count of $77(13-90)$ cells/ $/$ l. None of the patients had a CSF opening pressure measured at first LP. AMB was used as an induction agent in 51 (89\%) patients, of whom 47 (92\%) completed 2 weeks of AMB. Of these 51, only 20 (40\%) were prehydrated and $10(18 \%)$ had two repeat LPs performed 1 week apart. Renal function was monitored in only 27 (53\%) of the patients receiving AMB. This was done at baseline and twice weekly, and was consistent with the guidelines. No abnormality in renal function was recorded in these cases during the study. The mortality rate was $30 \%$ in the first 10 days of admission.

Conclusion. This chart review showed inadequate adherence to the recommendations of the 2007 SAHIVSoc guidelines in the majority of cases except for the use of AMB as a first-line antifungal agent. Control of ICP and monitoring for drug toxicity were not done as per guidelines and may impact on clinical care and outcome. Despite this, the early $30 \%$ mortality is comparable with published reports from other regions in SA, but is higher than in developed health systems.
\end{abstract}

S Afr J HIV Med 2014;15(3):104-107. DOI:10.7196/SAJHIVMED.991

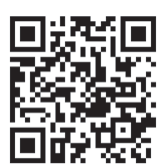

Worldwide, the burden of cryptoccocal menin gitis $(\mathrm{CM})$ is estimated to be $0.04-12 \%$ per year among persons with HIV, resulting in nearly

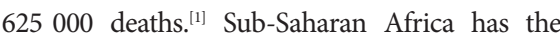
highest yearly burden with 720000 cases (range $144000-1.3$ million). ${ }^{[1]}$ In sub-Saharan Africa, mortality is estimated to be $50-70 \%$, compared with $12 \%$ in the USA and other developed nations. ${ }^{[2]}$ Cryptococcus neoformans infections are very rare in healthy people, with a yearly incidence of $0.4-1.3$ cases per 100000 in the general population. ${ }^{[2]}$ In a population-based surveillance of cryptococcosis in Gauteng, South Africa (SA), the overall incidence rate was 15.6/100 000 among both HIV-positive and -negative individuals. ${ }^{[3]}$ Among HIV-positive persons, the rate was 95/100 000, and among persons living with AIDS, 14/1 000. ${ }^{[3]}$

In HIV-positive individuals, CM may present with headaches, unexplained fever, nausea, vomiting, neck stiffness, confusion, seizures, abnormal behaviour, and new onset of psychiatric symptoms, altered level of consciousness, focal neurological signs, diplopia, unexplained blindness and sometimes coma. ${ }^{[4]}$ Headache is the predominant symptom and was observed in $100 \%$ of cases in a study done by Baradkar et al. ${ }^{[5]}$ Neck stiffness may be absent in some patients with $\mathrm{CM} \cdot{ }^{[6]}$ The presentation of $\mathrm{CM}$ may be acute, sub-acute (over 2 - 4 weeks), or chronic ( $>4$ weeks). ${ }^{[4]}$

Despite the availability of highly active antiretroviral treatment (HAART), the mortality from $\mathrm{CM}$ has not changed from the pre-HAART era. However, CM immune reconstitution inflammatory syndrome (IRIS) is implicated in the ongoing high mortality. ${ }^{[7]}$ It is also not uncommon to have other opportunistic infections together with CM that contribute to mortality. ${ }^{[8]}$

Elevated intracranial pressure (ICP) results in increased mortality and morbidity. Up to $40 \%$ of $\mathrm{CM}$ deaths occurring during weeks 3 - 10 of treatment were associated with elevated ICP. Visual loss was also observed as a consequence of elevated ICP. ${ }^{[9]}$

A high index of suspicion is needed for the diagnosis of CM, especially in sub-Saharan Africa, where there is a dearth of standard laboratory facilities and expertise. Therefore, the role 
of a detailed history and physical examination cannot be overemphasised. ${ }^{[10]}$ The definitive diagnosis involves cerebrospinal fluid (CSF) microscopy, cryptococcal antigen (CrAG) detection in CSF and/or serum, and the culture of C. neoformans. ${ }^{[1]]}$ If an Indian ink stain is negative, CrAG remains a reliable test $(95 \%$ sensitivity, 95\% specificity). The CrAG Latex Agglutination System (Meridien Bioscience, US) is commonly used and correlates well with the fungal burden. ${ }^{[11]}$

In a retrospective study conducted in Durban, SA, the significantly higher Indian ink positivity rate and significantly higher mean CrAG titre reflected the greater fungal load in HIV-positive patients ${ }^{[12]}$ Culture of C. neoformans in CSF is also beneficial in the diagnosis of subsequent episodes of CM where both CrAG and Indian ink tests are not useful. ${ }^{[13]}$

The only guideline for the management of CM in our setting, prior to 2012 when this study was conducted, was published by the SAHIVSoc in 2007. ${ }^{[6]}$ This guideline was updated in 2013. The initial guideline highlighted the role of amphotericin B (AMB) as the antifungal agent preferred during the induction phase of treatment. Treatment with AMB $(1 \mathrm{mg} / \mathrm{kg}$ body weight (BW)/day) for 2 weeks, followed by fluconazole $(400 \mathrm{mg} /$ day $)$ as a consolidation agent for 8 weeks was recommended. A dose of $200 \mathrm{mg}$ fluconazole daily is indicated for secondary prophylaxis until the $\mathrm{CD} 4^{+}$ T-lymphocyte count is $>200$ cells $/ \mu$ for at least 6 months and the most recent HIV-1 viral load is suppressed; this applies to patients with a first episode of $\mathrm{CM}^{\left[{ }^{[6]}\right.}$ Patients with relapse are treated with $1 \mathrm{mg} / \mathrm{kg}$ BW AMB for 2 - 4 weeks or until the CSF is sterile. Consolidation and maintenance options in relapse depend on the fluconazole minimum inhibitory concentration and include high-dose fluconazole with or without weekly $\mathrm{AMB}$ infusions, or voriconazole. ${ }^{[6]}$

$\mathrm{AMB}$ is often unavailable in our centres, hence high-dose fluconazole ( $800-1200 \mathrm{mg}$ ) is often used. The latter is suboptimal at these doses. ${ }^{[14]}$ For resource-limited settings where AMB is unavailable, high-dose fluconazole (1 $200 \mathrm{mg})$ is recommended as an induction agent by the World Health Organization (WHO) ${ }^{[15]}$

Similar to the SAHIVSoc guidelines, Tronconso et al. ${ }^{[16]}$ highlighted the role of monitoring renal function while patients are undergoing the induction phase with $\mathrm{AMB}$. If creatinine increases by twofold, the $\mathrm{AMB}$ dose is omitted and prehydration with $0.9 \%$ normal saline, 118 -hourly, is recommended. AMB should be stopped if the creatinine fails to decrease after this intervention. ${ }^{[6]}$
Table 1. Presenting symptoms and signs of patients admitted with cryptococcal meningitis $(N=57)$

\begin{tabular}{lll}
\hline Presenting complaints & $\boldsymbol{n}$ & \% \\
\hline Headache & 52 & 91 \\
Confusion & 30 & 53 \\
Psychotic features & 11 & 19 \\
Seizures & 13 & 23 \\
Vomiting & 26 & 46 \\
Signs & & \\
$\quad$ Fever & 29 & 51 \\
$\quad$ Neck stiffness & 34 & 60 \\
Kernig's and Brudzinski's signs & 34 & 60 \\
Focal signs (hemiparesis) & 1 & 2 \\
Cranial nerve deficit & 1 & 2
\end{tabular}

In June 2013, the SAHIVSoc updated the guideline for the prevention, diagnosis and management of CM in HIV-positive persons to be compatible with WHO guidelines. A major change from the 2007 version is the role of both $\mathrm{AMB}$ and fluconazole during the induction phase. The revision also highlighted timing of HAART initiation. ${ }^{[13]}$

\section{Methods}

The study was a descriptive, retrospective chart review conducted at the Mthatha General Hospital in the Eastern Cape (EC), SA, between December 2011 and May 2012. The majority of patients with $\mathrm{CM}$ in the OR Tambo district of the EC are managed as inpatients in this facility. The hospital serves as a referral centre and has 100 medical beds.

The inclusion criteria were adult patients $>18$ years of age with a first episode of CM diagnosed on lumbar puncture (LP) as part of routine care of patients with clinical features of meningitis. Patients were managed by a team of doctors that included an intern, medical officer/registrar and a family physician. The researcher did not interact with the patients.

For data collection, a researcher searched the ward register daily for cases diagnosed as CM and admitted to any of the medical wards. Data were collected from respective patient case note files. The medical records of all patients identified with CM from the ward register were available for data collection and were verified by positive laboratory results.

The data of interest were: measurements of CSF opening pressure at the first LP; whether $\mathrm{AMB} /$ fluconazole therapy was used; whether prehydration was administered before the receipt of AMB; and whether monitoring of renal function (rising urea and creatinine only) and ICP were done according to the recommendations of the SAHIVSoc 2007 CM guidelines.

The time of commencement of AMB was documented in the prescription/drug chart of each patient and the time of admission was recorded on the admission chart and the nurses' charts. The renal function of each patient was determined from the laboratory report in the case notes.

Data were analysed using SPSS version 18.0 (IBM, South Africa). Descriptive data were presented using frequency tables. Ethical approval was obtained from the Biomedical Research Ethics Committee of the University of KwaZulu-Natal (number BE058/12).

\section{Results}

A total of 57 charts were reviewed over the 6-month study period, representing all patients who were admitted to the medical wards with CM in this time period. However, some patients may have died in casualty before ward admission and hence would not have been captured in the ward admission charts. The mean age (range) of the patients was 36 (21 - 60) years, and 40 were women (70\%). Table 1 shows the presenting complaints, signs and their frequency of occurrence. Of the 57 patients, 52 (91\%) presented with headache. Confusion was recorded in $30(53 \%)$ and vomiting in $26(46 \%)$ of subjects. The major signs observed were fever in $29(51 \%)$, and meningeal signs in 34 (60\%).

All patients had an initial LP for CSF laboratory analysis that included biochemistry, Indian ink stain, CrAG assay and fungal culture. A computerised tomography (CT) scan was done in only two patients with 
Table 2. Concurrent opportunistic infections and comorbidities in patients with cryptococcal meningitis $(\boldsymbol{N}=57)$

\begin{tabular}{lll}
\hline Opportunistic infections & $\boldsymbol{n}$ & \% \\
\hline Pulmonary tuberculosis & 32 & 56 \\
Oropharyngeal candidiasis & 57 & 100 \\
Seborrhoeic dermatitis & 12 & 21 \\
Oral hairy leukoplakia & 8 & 14 \\
Tuberculous meningitis & 10 & 23
\end{tabular}

focal signs and cranial nerve deficits, showing a mass lesion in both cases, suggestive of cryptococcoma. There was no record of the measurement of CSF opening pressure at LP in any of the charts reviewed. The diagnosis of CM was made within 24 hours of presentation (range 3 - 8 hours) in $70 \%$ of the patients. This was calculated from the time the LP was performed to the time the microscopy result was obtained. All 57 cases were positive for microscopy and culture for C. neoformans, while CrAG was positive in 51 (89\%) cases.

HIV infection was diagnosed for the first time in 7 (12\%) of these patients. The remaining 50 (88\%) were known to be HIV-positive. All had features of immunosuppression. The most frequent infections were oropharyngeal candidiasis and tuberculosis (TB) (Table 2).

The median (range) $\mathrm{CD} 4^{+}$count was 77 (51 -

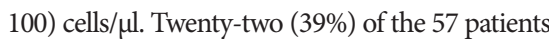
were on HAART. Thirteen (23\%) patients had been on ARVs for less than 6 months and the remaining 9 (16\%) patients had been on ARVs for 7 - 12 months. All patients were on tenofovir, lamivudine and efavirenz and were documented to be adherent to treatment. Tenofovir was continued even when $\mathrm{AMB}$ was commenced.

Thirty-two (56\%) of the patients had co-infection with pulmonary TB. Ten of these patients were diagnosed on sputum microscopy for acid-fast bacilli, while the remainder were diagnosed clinically and on chest radiography. Ten patients had tuberculous meningitis co-infection based on CSF lymphocytosis and raised protein.

$\mathrm{AMB}(1 \mathrm{mg} / \mathrm{kg} \mathrm{BW})$ was used as the induction agent for the management of CM in 51 (89\%) of the patients, while the remaining $6(11 \%)$ had intravenous fluconazole ( $800 \mathrm{mg}$ ) because AMB was out of stock. Among the 51 patients who had $\mathrm{AMB}$ as an induction agent, the drug was initiated in 50 (98\%) cases, within 24 hours of the attending doctor writing the script. The time of commencement of AMB was documented in the prescription/drug chart by the nurse administering the dose and the time of admission was recorded on the patient chart. Only 20 (39\%) patients receiving $\mathrm{AMB}$ had documentation of adequate prehydration. Forty-seven (92\%) patients completed the 2-week course of AMB.

Renal function was monitored in 27 (53\%) of the patients receiving AMB. This was done at baseline and twice weekly, and was consistent with the guideline. Results were obtained from the case files and the normal ranges for urea and creatinine were $2.1-7.1 \mathrm{mmol} / \mathrm{l}$ and 64 $104 \mu \mathrm{mol} / \mathrm{l}$, respectively. No abnormalities in serum urea and creatinine were recorded in these cases during the study.

Ten patients (18\%) who experienced worsening headache in the ward had two repeat LPs performed for the management of raised ICP. These LPs were performed 1 week apart.

Forty patients (70\%) were discharged, with the average (range) hospital stay being 21 (16 28 ) days. The in-hospital mortality rate within the first 1 - 10 days of hospital admission was $30 \%$. Fluconazole $400 \mathrm{mg}$ was dispensed on discharge for 1 week of the consolidation phase and patients were referred to a local clinic to continue treatment. Data on patients prescribed HAART were not obtained.

\section{Discussion}

There are few data on management and outcome of CM from a non-research district hospital setting in a resource-limited health system where operational challenges may have a significant impact. This study was limited by the retrospective, descriptive nature. Although all patient charts were found, documentation in the clinical notes was poor and missing data compromised this chart review.

The Mthatha complex manages 6 - 10 cases of CM in AIDS patients per month. Some cases of CM could have been missed in this review if patients died in casualty and hence were not captured in the ward admission file. This retrospective chart review of adult patients found that the management was not consistently in line with national guidelines. Unlike other studies where the incidence of $\mathrm{CM}$ was similar in both sexes, or higher in men, ${ }^{[1]}$ we found that the disease was three times higher in women. The reason for this may be the migrant nature and different health-seeking behaviour of men in this community and warrants further investigation. All patients were $\mathrm{HIV}$-positive with a median $\mathrm{CD} 4^{+}$ count of 77 cells $/ \mu \mathrm{l}$, consistent with the results in other studies. ${ }^{[1,3]}$ An interesting observation is that $88 \%$ of patients were known to be infected with HIV prior to the diagnosis of CM. The mortality of $30 \%$ was less than that reported in a Ugandan study in a similar patient population and healthcare system, where mortality was between $40 \%$ and $50 \% .{ }^{[7]}$ The mortality was similar to that in other centres in SA. ${ }^{[17]}$

Many patients already diagnosed with HIV infection were not on ARVs at the time of presentation. This is of concern as it is likely that CM may have been preventable in the majority. This observation highlights the need for a more intensive rollout of ARV treatment. Our study also highlighted the occurrence of CM in the first 6 months of HAART, which may represent IRIS or reflect ongoing immunosuppression during early HAART.

Headache was the most common symptom. Vomiting, fever, confusion and psychosis were also noted. However, meningeal signs were absent in $40 \%$ of the patients. This may be owing to poor inflammatory response attributed to severe immunosuppression.

The study found that co-infections, particularly $\mathrm{TB}$, were extremely high in these $\mathrm{CM}$ patients. This is not surprising, because of profound immunosuppression and the high prevalence of TB in our setting. Many cases of TB were diagnosed on clinical or radiological features, or suggestive CSF microscopy and biochemistry. Since these are not specific diagnostic tests, overdiagnosis of TB was possible. The CSF CrAG was found to be an extremely important test; $77 \%$ of the patients were diagnosed within 24 hours of presentation using this assay. The CrAG test should be routinely performed by the laboratory if the India ink stain is negative. The effect of early diagnosis on morbidity and mortality is well documented. ${ }^{[18]} \mathrm{It}$ was also demonstrated that patients who presented with features of neurological deficit received a CT scan as recommended by the guideline. ${ }^{[6]}$ None of the patients admitted had an opening CSF pressure measured during the LP and fundoscopy was not done, which is of concern. Documentation and management of ICP was also not done. This was presumably because the hospital does not stock manometers.

Drug treatment aspects of the 2007 SAHIVSoc guidelines were followed in most 
patients who received $\mathrm{AMB}$ as the induction agent, and all were discharged on fluconazole, $400 \mathrm{mg}$ daily, as a consolidation agent. ${ }^{[6]}$

Prehydration and renal toxicity monitoring advised by the guideline were not followed, and it is surprising that more renal complications were not observed. However, documentation of fluid administration is poor in our facility. Early mortality rates may have improved if these measures had been implemented.

\section{Conclusion}

We recommend that an in-service workshop on common opportunistic infections in HIV should be provided for healthcare providers within the district hospital setting. A commitment by the Department of Health to provide necessary diagnostic tools, including manometers, and an uninterrupted drug supply would improve the management and outcome of the disease at a district level.

\section{References}

1. Park BJ, Wannemuehler KA, Marston BJ, Govender N, Pappas PG, Chiller TM. Estimation of the current global burden of cryptococcal meningitis among persons living with HIV AIDS. AIDS 2009;23(4):525-530. [http://dx.doi.org/10.1097/QAD.0b013e328322ffac]

2. Centers for Disease Control and Prevention. C. neoformans cryptococcosis. http//:www. cdc.gov/fungal/crptococcosis-neoformans/statistics.html (accessed 27 July 2013).

3. McCarthy KM, Morgan J, Wannemuehler KA, et al. Population-based surveillance for cryptococcosis in an antiretroviral-naive South African province with a high HIV seroprevalence. AIDS 2006;20(17):2199-2206. [http://dx.doi.org/10.1097/ QAD.0b013e3280106d6a]

4. Bicanic T, Harrison TS. Cryptococcal meningitis. Br Med Bull 2004;72(1):99-118. [http://dx.doi.org/10.1093/bmb/ldh043]

5. Baradkar V, Mathur M, De A, Kumar S, Rathi S. Prevalence and clinical presentation of cryptococcal meningitis among HIV seropositive patients. Indian J Sex Transm Dis 2001;30(1):19-22. [http://dx.doi.org/10.4103/0253-7184.55474]

6. McCarthy K, Meintjes G, Arthington-Skaggs B, et al. Guidelines for the prevention, diagnosis and management of cryptococcal meningitis and disseminated cryptococcosis in HIV-infected patients. S Afr J HIV Med 2008;3(25):25-35. http:// sajhivmed.org.za/index.php/sajhivmed/article/view/92 (accessed 6 June 2013).

7. Kambugu A, Meya D, Rhein J, et al. Outcomes of cryptococcal meningitis in Uganda before and after the availability of HAART. Clin Infect Dis 2008;46(11):1694-1701. [http://dx.doi.org/10.1086/587667]

8. Skiest DJ, Hester LJ, Hardy RD. Cryptococcal immune reconstitution inflammatory syndrome: Report of four cases in three patients and review of the literature. J Infect 2005;51(5): e289-e297. [http://dx.doi.org/10.1016/j.jinf.2005.02.031]

9. Van der Horst CM, Saag MS, Cloud GA, et al. Treatment of cryptococcal meningitis associated with the acquired immunodeficiency syndrome. National Institute of Allergy and Infectious Diseases Mycoses Study Group and AIDS Clinical Trials Group. N Engl J Med 1997;337(1):15-21.

10. Lightowler JVJ, Cooke GS, Mutevedzi P, et al. Treatment of cryptococcal meningitis in KwaZulu-Natal, South Africa. PLoS ONE 2010;5(1):e8630. [http://dx.doi. org/10.1371/journal.pone.0008630]

11. Tanner DC, Weinstein MP, Fedorciw B, Joho KL, Thorpe JJ, Reller L. Comparison of commercial kits for detection of cryptococcal antigen. J Clin Microbiol 1994;32(7):16801684 .

12. Moosa MYS, Coovadia YM. Cryptococcal meningitis in Durban, South Africa: A comparison of clinical features, laboratory findings, and outcome for human immunodeficiency virus (HIV)-positive and HIV-negative patients. Clin Infect Dis 1997;24(2):131-134.

13. Southern African HIV Clinicians Society. The Guideline for the Prevention, Diagnosis and Management of Cryptococcal Meningitis among HIV-infected Persons: 2013 Update. S Afr J HIV Med 2013;2(4):76-86. [http://dx.doi.org/10.7196/sajhivmed.930]

14. Pukkila-Worley R, Mylonakis E. Epidemiology and management of cryptococcal meningitis: Developments and challenges. Expert Opin on Pharmacother 2008;9(4):551-560. [http://dx.doi.org/10.1517/14656566.9.4.551]

15. World Health Organization. Rapid advice: Diagnosis, prevention and management of cryptococcal disease in HIV-infected adults, adolescents and children. Geneva: World Health Organization, 2011:4-44. http://whqlibdoc.who.int/ publications/2011/9789241502979_eng.pdf (accessed 2 January 2014).

16. Troncoso A, Fumagalli J, Shinzato R, Gulotta H, Toller M, Bava J. CNS cryptococcoma in an HIV-positive patient. J Int Assoc Physicians AIDS Care (Chic) 2002;1(4):131-133

17. Lessells RJ, Mutevedzi PC, Heller T, Newell ML. Poor long-term outcomes for cryptococcal meningitis in rural South Africa. S Afr Med J 2011;101(4):251-252.

18. Dhana A. Diagnosis of cryptococcosis and prevention of cryptococcal meningitis using a novel point-of-care lateral flow assay. Case Reports in Medicine 2013;2013(Article ID 640216):4 pp. [http://dx.doi.org/10.1155/2013/640216] 